\title{
A bizarre highly vascular tumor with alarming presentation: a diagnostic dilemma
}

\author{
Kamal Buckshee ${ }^{1 *}$, Rastogi Harsh ${ }^{2}$, Arora Deepshikha ${ }^{3}$, Rohatgi B. Tanya ${ }^{1,4}$
}

\author{
${ }^{1}$ The woman Clinic, Noida, Uttar Pradesh, India \\ ${ }^{2}$ Department of Radiology and Intervention, Indraprastha Apollo Hospitals, Delhi, India \\ ${ }^{3}$ Department of Cellular Pathology, Leeds Teaching Hospitals, St. James's University Hospital, Beckett Street, Leeds, \\ LS9 7TF, UK \\ ${ }^{4}$ Department of Reproductive Medicine and Assisted Reproductive Techniques (IVF), Max Medcentre and Max Super \\ Speciality Hospital, Delhi, India
}

Received: 02 October 2017

Accepted: 31 October 2017

\author{
*Correspondence: \\ Dr. Kamal Buckshee, \\ E-mail: kamalbuckshee@gmail.com
}

Copyright: (c) the author(s), publisher and licensee Medip Academy. This is an open-access article distributed under the terms of the Creative Commons Attribution Non-Commercial License, which permits unrestricted non-commercial use, distribution, and reproduction in any medium, provided the original work is properly cited.

\begin{abstract}
An exceedingly rare, highly vascular tumor with atypical and alarming presentation posed diagnostic and management challenges. A 29 years old lady, complained of abdominal and pelvic pain, nausea, vomiting and repeated spells of fainting attacks following the jumping episode (2-3 times). Emergency admission and investigations at United States of America (USA) revealed a large, complex, highly vascular, irregular mass displaying finger like projections, filling the lower abdomen, pelvis and large amount of intra peritoneal bleed. A provisional diagnosis of arteriovenous malformation (AVM), arteriovenous fistula (AVF), and rupture/leak was made. Uterine artery angiography (UAA), preoperative embolization and possibility of hysterectomy as a life saving measure were suggested, to which the couple declined and got discharged. To save uterus and fertility they presented to us for second opinion and further management. Angiography, magnetic resonance imaging (MRI) and preoperative uterine artery embolization were done followed by exploratory laparotomy which revealed hemoperitoneum and a large weird looking vascular tumor (a veil of sea weeds/dark purple nodules of various sizes resembling cotyledons of placenta). Intra-operative tissue analysis (frozen section) suggested benign pathology. Mass was removed completely and fertility sparing surgery was undertaken. Histopathological diagnosis was cotyledonoid dissecting leiomyoma (CDL). No recurrence/metastasis has been observed on follow up of 5 years.
\end{abstract}

Keywords: Cotyledonoid dissecting leiomyoma, Uterine artery angiography, Uterine artery embolization.

\section{INTRODUCTION}

A bizarre tumor with unusual growth pattern, highly vascular, infiltrative character, resembling placenta has been recognized and named cotyledonoid dissecting leiomyoma (CDL) or Sternberg tumor (ST). ${ }^{1}$

It is an unusual variant of uterine leiomyoma and its gross features mimic malignancy. Smith et al summarized clinical, radiological and pathological features of 41 previously reported cases. ${ }^{2}$ At present 43 cases have been reported. ${ }^{3}$ Our aim is to highlight a strange and alarming presentation of a unique tumor which added further confusion to the existing diagnostic puzzle.

To the best of our knowledge, probably this would be the first case to be reported with an unusual clinical manifestation. 


\section{CASE REPORT}

A 29-year-old nulligravida (no contraception, seeking pregnancy) complained of abdominal and pelvic pain, nausea, vomiting and repeated spells of dizziness following the jumping episode (2-3 times) to pick up articles from the top shelf. Investigations at USA revealed a large, complex, highly vascular mass with irregular borders, filling the lower abdomen, pelvis and large amount of intra peritoneal bleed. Uterine artery angiography to clinch diagnosis and therapeutic surgery with risk of hysterectomy as a life saving measure were suggested, but the couple was not willing to undergo any surgery with a possible risk of hysterectomy, so she got discharged and presented to us for further management.

Menarche at 11 years. Cycles were regular with normal flow. Obstetric history: nulligravida. History: Patient was asymptomatic till 2007, but started having intermittent, abdominal pain, associated with nausea, vomiting and occasional episodes of giddiness (2008). Sonography and MRI (2009-India) revealed a large exophytic mass $(10 \times 8 \times 4.5 \mathrm{~cm})$, solid, heterogeneous, hypo-echoic occupying the whole of pelvis, inseparable from the uterus and highly vascular.

Both ovaries were normal and there was no free fluid in pelvis. A diagnosis of subserosal fibroid was made. Conservative management was adopted as the tumor was benign and she was unmarried. In 2011 she got married and moved to USA. Since her symptoms persisted, sonography with color doppler (CD), computed tomography (CT) abdomen and pelvis were done (March 2012) which indicated right adnexal mass with dilated and thrombosed pelvic veins.

In July 2012, she experienced abdominal and pelvic pain, nausea, vomiting and repeated spells of fainting attacks following the jumping episode. Emergency admission and investigations at USA (July 2012), trans-abdominal (TA), trans-vaginal ultrasound (TVS) with CD, CT and MRI (abdomen and pelvis) revealed a large, complex, irregular mass, $14.6 \times 7.3 \times 14.1 \mathrm{~cm}$, predominantly solid with some cystic components anterior to the uterus inseparable from the myometrium. It was highly vascular, displaying finger like/serpiginous/frond like projections with prominent vessels in the centre of the fronds, some of which were seen to float in the free fluid, filling the lower abdomen, pelvis and large amount of hemoperitoneum.

Both ovaries were enlarged, polycystic and there was endometrioma in the right ovary. Her hematocrit dropped from 34 to 27. A provisional diagnosis of AVM/AVF with rupture/leak, angiosarcoma, endometriosis, leiomyoma with haemorrhagic degeneration were considered. In view of intra-abdominal bleed from a highly vascular tumor, she and her husband were advised and counselled for UAA to clinch diagnosis and therapeutic surgery with risk of hysterectomy as a life saving measure. The couple understood that delaying diagnostic/therapeutic surgery was dangerous to wife's health, but they did not want to take any risk of hysterectomy, even in the face life threatening bleeding. In view of intra-peritoneal bleed, risk of severe bleeding, embolism and other complications they were advised not to travel. But they still travelled to seek second opinion. They presented to us for further evaluation and management.

\section{Examination}

She looked pale and anxious, her vitals were stable, abdomen was distended, slightly tender and a vague mass was felt in the lower abdomen. A gentle abdominal/pelvic examination revealed normal uterus with fullness in all the fornixes. Sonography with CD, hemogram, thalassemia screen, rubella $\operatorname{IgG}$ and $\mathrm{IgM}$, Beta-human chorionic gonadotropin ( $\beta \mathrm{HCG})$, antibody screen, PT, APTT, CA125, kidney/thyroid function, lipid profile, fasting blood sugar and uterine artery angiography were performed to establish the diagnosis, asses her current status and to plan surgical treatment.

Ultrasound (US) revealed a large, highly vascular mass, engulfing the uterus anteriorly, laterally, posteriorly and along the fundal region and free fluid in pelvis. HIFU screening and MRI indicated that there was no AVF or AVM but a possibility of a highly vascular leiomyoma and CA125 was $79.30 \mathrm{u} / \mathrm{ml}$. When the intra-peritoneal hemorrhage decreased CA125 level dropped to 40.60 $\mathrm{u} / \mathrm{ml}$. She had beta thalassemia minor and hemoglobin $(\mathrm{Hb})$ was $9.2 \mathrm{gm} / \mathrm{dl}$, PCV 29.2.

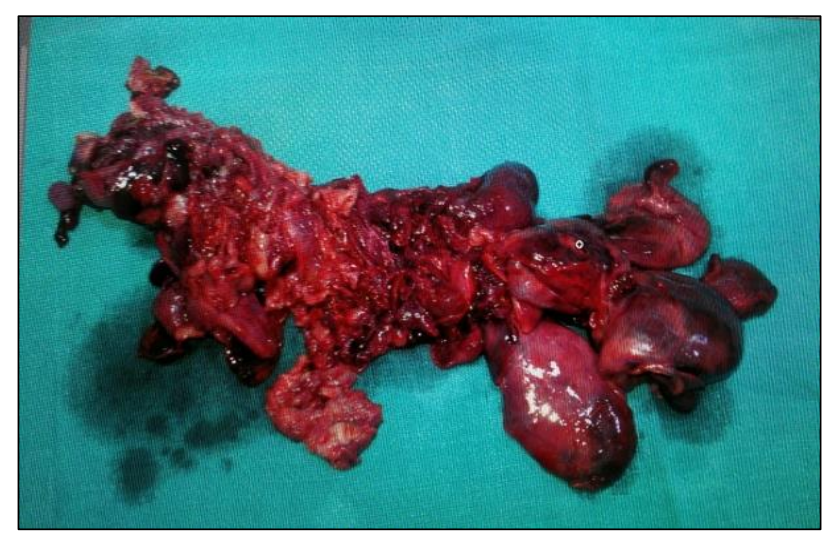

Figure 1: Gross appearance of the resected exophytic mass $(26 \times 24 \times 10 \mathrm{~cm})$ reveals nodularity and filiform appearance and innumerable, congested, bulbous (cotyledonoid) nodules, varying from 0.5 to $3.0 \mathrm{~cm}$ in their greatest dimension.

Angiography revealed highly vascular tumor supplied by both uterine and ovarian arteries. In view of above findings, preoperative uterine artery embolization (UAE) was performed and vascularity of the tumor decreased by $90 \%$. The couple was counselled and informed consent for hysterectomy was taken. Exploratory laparotomy 
revealed hemoperitoneum. Dark bluish/dark purple color nodules (angionodular) of varying sizes $(0.5$ to $3.0 \mathrm{~cm})$ like bunch of black grapes/cotyledons of a placenta attached to each other with thin, flimsy, fragile strands of dilated vessels occupying the lower abdomen, whole of pelvis engulfing the uterus all over, attached to the lateral pelvic walls, anteriorly to the bladder, posteriorly to the sacrum and to the omentum. However, no nodules were seen in the omentum. These nodules looked like dark reddish sea weeds floating in the haemorrhagic fluid in peritoneal cavity.

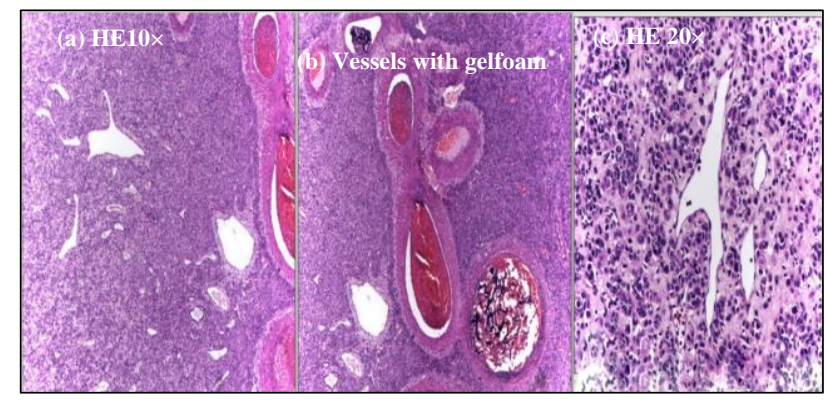

Figure 2: (a) $10 \times$ magnification, hematoxylin and eosin stain (H and $\mathbf{E})$. The tumor is cellular and composed of sheets of round cells supported by rich vascularity. (b) Many thick walled congested vessels are seen with some showing gelfoam used for embolization. (c) $\mathrm{H}$ and $\mathrm{E} 20 \times$ the tumor cells are uniform, large, round to epithelioid.

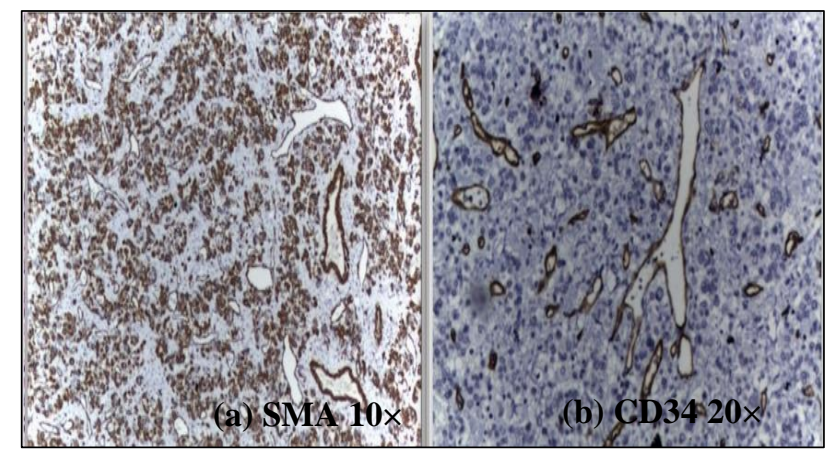

Figure 3: (a) SMA 10× the tumor cells are uniform, large, round to epithelioid and show strong diffuse positivity with SMA. (b) CD34 20× however, the tumor cells are negative with $\mathrm{CD34}$, but the rich vasculature is highlighted by CD34.

Peritoneal washings were sent for cytology. The mass was separated from both lateral pelvic walls, anteriorly and posteriorly. It appeared to be arising from the right anterior wall of the uterus near the cornu by a small pedicle. Uterus was very soft, no fibroid could be felt or seen, mass was removed completely along with the pedicle and superficial part of the myometrium of the uterus. Homeostasis was secured. Right ovary (RO) had endometriotic implant which was fulgurated, left ovary (LO) was normal. 2 units of packed red blood cells (PRC) were given. Frozen section revealed benign CDL.
Postoperative period was uneventful. Histopathological diagnosis was mesenchymal tumor with smooth muscle differentiation and cotyledonoid leiomyoma (Figure 1, 2 and 3). Clinical follow up with abdominal and pelvic examination along with US of the abdomen and pelvis revealed normal uterus, polycystic ovaries, CA125 was $19.4 \mathrm{u} / \mathrm{ml}$ and after 3 months of surgery MRI of the abdomen revealed no mass adjacent to the anterior wall of the uterus and no definite residual tumor.

\section{DISCUSSION}

Cotyledonoid dissecting leiomyoma (CDL) is a distinct entity and an extremely rare tumor. It has a dissecting nature towards the myometrium and the extra uterine part protrudes in to the pelvic cavity that resembles the cotyledons of the placenta. ${ }^{1}$

Menolscino et al named this tumor "angionodular dissecting leiomyoma (ADL)". 4 Reviewed literature indicates that age distribution ranges from 23-73 years and presents either with a pelvic mass, abnormal uterine bleeding, abdominal pain, bloating, constipation, utero vaginal prolapse or they could be asymptomatic. ${ }^{2}$ Our patient was 29 years, nulliparous and complained of abdominal pain, nausea, vomiting and sometimes, giddiness off and on and at times during menses since 2008. She experienced acute abdominal pain associated with fainting attacks and had large intra peritoneal bleed following the jumping episode (2012). None of the cases reported so far have presented with above mentioned complaints and or intra-peritoneal bleed. Abdominal pain, associated with fainting spells which could probably be due to twists/torsion/micro thrombi of thin fragile vessels of the extra uterine part that might have occurred following minor trauma/jumping/jerky movement. Whereas abdominal pain, fainting spells and intra peritoneal bleed could have resulted either due to rupture/leak of thin fragile vessels of the extra uterine part protruding in to the pelvic cavity following the jumping episode.

Sonography with CD, CT and MRI are excellent modalities to clinch the diagnosis provided one is aware of CDL and its findings on US/MRI. In our case sonography and MRI (2009) suggested a highly vascular leiomyoma. In March 2012, CD and CT revealed thrombosis and dilatation of pelvic veins indicating that these vessels are prone to thrombosis. The US and MRI findings (July 2012) were similar to our intra-operative findings. But diagnosis of CDL was not entertained due to the lack of awareness and rarity of the tumor. Presence of vascular leiomyoma since 2009 to 2012 indicated that tumor was probably benign. However, atypical presentation and intra-peritoneal bleed confused the gynecologist, radiologist and surgeons and thus diagnosis of CDL was over looked. Findings of US, MRI (USA 2012) and review of literature made us aware and suspicious of CDL and we requested for intra-operative tissue analysis. An accurate diagnosis is essential prior to 
surgery especially in young women desirous of pregnancy for optimum management. Preda et al reported MRI findings of a case of CDL. ${ }^{5}$ Only 10 cases of CDL were reported till 2007, its number increased to 20 in $2011 .^{6}$ Awareness and suspicion of this entity increased its number to 43 in $2016 .^{3}$ Intense desire to save uterus and fertility made our young, nulliparous patient restless to undertake any risk at any cost.

Intra-operative tissue analysis (frozen section) suggested benign pathology so we performed fertility sparing/preserving surgery. The best treatment for CDL is surgery. It is reported to be a benign tumor without the potential for metastasis or recurrence and has good prognosis. However, recently a case of recurrence following myomectomy has been reported. ${ }^{7}$ Incomplete resection of the tumor may lead to recurrence. Patients with exophytic component contiguous with the intramural components of the uterus should be followed clinically and by US yearly.

\section{CONCLUSION}

Awareness and suspicion of CDL is essential among obstetricians, gynaecologist, surgeons, radiologist and pathologists for early diagnosis and optimum management. Intra-operative frozen section is crucial to avoid over treatment and fertility sparing surgery to be done in women desiring pregnancy.

This case highlights a rare and alarming presentation posing diagnostic and management dilemma. It also highlights that thin, fragile, dilated vessels are prone to torsion/constriction/rupture/leak following jerks/trauma and are susceptible to thrombosis.

The intense desire of a young nulliparous woman to save the uterus and fertility at any cost reveals the significance of fertility sparing surgery in women desiring pregnancy.
Funding: No funding sources Conflict of interest: None declared Ethical approval: Not required

\section{REFERENCES}

1. Roth LM, Reed RJ, Sternberg WH. Cotyledonoid dissecting leiomyoma of the uterus: the Sternberg tumor. Am J Surg Pathol. 1996;20(12):455-61.

2. Smith CC, Gold MA, Wile G, Fadare O. Cotyledonoid dissecting leiomyoma of the uterus: a review of clinical, pathological, and radiological features. Int J Surg Pathol. 2012;20(4):330-41.

3. Xu T, Wu S, Yang R, Zhao L, Sui M, Cui M, et al. Cotyledonoid dissecting leiomyoma of the uterus: a report of four cases and a review of the literature. Oncology Letters. 2016;11(4):2865-8

4. Menolascino-Brrata F, de Barriola GV, de Gomez NM, Tamayo GJ, Suarez JA, Chacon HAV. Cotyledonoid dissecting leiomyoma (Sternberg tumor): an unusual form of leiomyoma. Pathol Res Pract. 1999;195(6):435-8.

5. Preda L, Rizzo S, Gorone MS, Fasani R, Maggioni A, Bellomi M. MRI features of cotyledonoid dissecting leiomyoma of the uterus. Tumori. 2009;95(4):532-4.

6. Weissferdt A, Maheshwari MB, Downey GP, Rollason TP, Ganesan R. Cotyledonoid dissecting leiomyoma of the uterus: a case report. Diagn Pathol. 2007;2:18.

7. Roth LM, Kirker JA, Insull M, Whittaker J. Recurrent cotyledonoid dissecting leiomyoma of the uterus. Int J Gynecol Pathol. 2013;32:215-20.

Cite this article as: Buckshee $\mathrm{K}$, Harsh R, Deepshikha A, Tanya RB. A bizarre highly vascular tumor with alarming presentation: a diagnostic dilemma. Int J Reprod Contracept Obstet Gynecol 2017;6:5621-4. 